\title{
Single and molecular ion irradiation-induced effects in GaN : experiment and cumulative MD simulations
}

\section{Karaseov, P. A.}

2017-12-20

Karaseov , P A , Karabeshkin , K V , Titov , A I , Ullah , M W , Kuronen , A , Djurabekova , F , Nordlund, K , Ermolaeva , G M \& Shilov , V B 2017 , ' Single and molecular ion irradiation-induced effects in GaN : experiment and cumulative MD simulations ' , Journal of Physics. D, Applied Physics, vol. 50 , no. 50 , 505110 . https://doi.org/10.1088/1361-6463/aa97ab

http://hdl.handle.net/10138/308774

https://doi.org/10.1088/1361-6463/aa97ab

cc_by_nc_nd

acceptedVersion

Downloaded from Helda, University of Helsinki institutional repository.

This is an electronic reprint of the original article.

This reprint may differ from the original in pagination and typographic detail.

Please cite the original version. 
Preprint of paper published as Journal of Physics. D, Applied Physics. 50 (2017) 505110.

Single and molecular ion irradiation-induced effects in GaN: Experiment and cumulative MD simulations

\author{
P.A.Karaseov ${ }^{1}$, K.V.Karabeshkin ${ }^{1}$, A.I.Titov ${ }^{1}$, \\ Mohammad W. Ullah ${ }^{2,4}$, A. Kuronen ${ }^{2}$, F. Djurabekova ${ }^{2}$, K. Nordlund ${ }^{2}$, \\ G.M.Ermolaeva ${ }^{3}$, V.B.Shilov ${ }^{3}$ \\ ${ }^{1}$ Department of Physical Electronics, Peter the Great St.Petersburg Polytechnic University,29 \\ Polytechnicheskaya st., St. Petersburg, Russia \\ ${ }^{2}$ Department of Physics, University of Helsinki, P.O. Box 43, FIN-00014, Finland \\ ${ }^{3}$ Vavilov State Optical Institute, 12 Birzhevaya line, St. Petersburg, Russia \\ ${ }^{4}$ Materials Science and Technology Division, Oak Ridge National Lab, Oak Ridge, TN 37831, \\ USA
}

An investigation of mechanisms of enhancement of irradiation-induced damage formation in $\mathrm{GaN}$ under molecular in comparison to monatomic ion bombardment is presented. Ionimplantation-induced effects in wurtzite $\mathrm{GaN}$ bombarded with $0.6 \mathrm{keV} / \mathrm{amu} \mathrm{F}, \mathrm{P}, \mathrm{PF}_{2}$, and $\mathrm{PF}_{4}$ ions at room temperature are studied experimentally and by cumulative MD simulation in the correct irradiation conditions. In the low dose regime, damage formation is correlated with a reduction in photoluminescence decay time, whereas in the high dose regime, it is associated with the thickness of the amorphous layer formed at the sample surface. In all the cases studied, a switch to molecular ion irradiation from bombardment by its monatomic constituents enhances the damage accumulation rate. Implantation of heavy Ag ion, having approximately the same mass as the $\mathrm{PF}_{4}$ molecule, is less effective in surface damage formation, but leads to noticeably higher damage accumulation in the bulk. . The cumulative MD simulations do not reveal any significant difference in the total amount of both point defects and small defect clusters produced by light monatomic and molecular ions. On the other hand, increased production of large defect clusters by molecular $\mathrm{PF}_{4}$ ions is clearly seen in the vicinity of the surface. Ag ions produce almost the same number of small, but more large defect clusters compare to the others. This findings show that the enhancement of stable damage formation in $\mathrm{GaN}$ under molecular, as well as under heavy monatomic ion irradiation, can be related to the higher formation probabilityof large defect clusters.

\title{
1. Introduction
}

Gallium nitride $(\mathrm{GaN})$ is a wide bandgap $(3.4 \mathrm{eV})$ semiconductor with some superior properties compared to $\mathrm{Si}$ and GaAs $[1,2]$. It has attracted considerable interest due to the wide range of applications in high power electronics, microwave and optoelectronic devices, including the solidstate white lamps now replacing the traditional incandescent lamps due to much better efficiency [3-5]. In GaN-based device fabrication, ion bombardment is an attractive choice due to high precision and uniformity of dopant distribution, possibility of dry etching [4], electrical isolation $[4,7]$ and carrier and PL lifetime tuning techniques [8]. Ion irradiation is always accompanied by production of lattice disorder which affects all properties of the materials [9]. Hence, to understand fundamental processes and to improve device performance, it is vital to study irradiation-related defect formation.

Irradiation induced damage accumulation in semiconductors has been extensively studied for over half of a century. Despite the mature age of this research field, damage evolution is more or less well understood only for a few semiconductors ( $\mathrm{Si}$ and $\mathrm{GaAs})[9,10]$. This is related to the nonequilibrium nature of radiation effects, the complexity of various defect interaction processes and to the fact that solids can behave very diverse ways under ion bombardment. Particularly, damage 
Preprint of paper published as Journal of Physics. D, Applied Physics. 50 (2017) 505110.

accumulation under ion irradiation has a very specific behavior in gallium nitride. Even though it has been studied by several different research groups (see, for instance, [11-17]), many physical aspects of damage formation in GaN are still far from being understood. One of the existing problems is the influence of the density of an individual collision cascade on effectiveness of stable damage accumulation [17], which in turn gives rise to another irradiation-related phenomenon, the so-called molecular effect (ME) in stable damage accumulation. The latter effect can be observed by the comparison of lattice disorders (or other radiation-induced phenomena) produced by the same amount of atoms, either as a molecular ion or as separated monoatomic ions, provided by molecular and monoatomic ions having the same velocities, as well as a similar average fluence of atoms on the surface. In our case, the ME means that the molecular/cluster ions create more stable damage than the same number of constituent atoms implanted into the target separately. This effect has long been known and was studied in numerous investigations (see, for instance, [18-26] and references therein, as well as reviews of earlier publications [27, 28]). Most of these works were focused on investigation of the ME during ion implantation of $\mathrm{Ge}$ (earlier publications) and $\mathrm{Si}$. A number of experimental studies of the ME was performed also for ion irradiation of $\mathrm{GaN}$ [17, 29 - 30], as well as other semiconductor materials. The results have confirmed the manifestation of ME in a wide range of bombardment conditions of semiconductors by molecular ions, which motivates a detailed investigation of the mechanisms of this phenomenon.

If the correct irradiation conditions are used, the only difference between monatomic and molecular ion bombardment is that atomic ions hit the surface at randomly distributed positions, but atoms comprising a molecular ion all together practically hit at the same point. Thus, collision cascades generated by the atomic components of a molecular ion will overlap in the initial part of their trajectories. As a result, the density of displacements in the averaged individual collision cascade in the surface vicinity increases [17]. Increase in the cascade density leads to (i) a superlinear primary defect generation (an energy spike effect) [28], and (ii) an enhancement of secondary defect formation $[21,22]$.

However, another additional reason of increased stable defect formation efficiency in a dense cascade is possible. It has been shown earlier [32] by MD simulation that irradiation of GaAs and GaAsN by heavy ions leads to formation of larger defect cluster than in case of light ion bombardment. In our previous work [33], MD simulations also showed that in the case of bombardment of $\mathrm{GaN}$ by both molecular and monatomic heavy ions, the increase in displacement density leads to an enhancement of larger defect cluster formation.

In practice, however, the experimentally measured damage is formed by several ions sequentially stopped in the same region, which was not considered in earlier studies. Thus, the aim of the present work is to understand whether the effect of enhancement of the formation of large defect clusters observed in MD simulation of single impacts results also at higher fluences in a sequential disordering of $\mathrm{GaN}$ by molecular and heavy ions.. Another goal of the present MD research is to investigate the dependence of such disordering on ion fluence. Finally, it was predicted in [33] that such large defect clusters are responsible for the decay of photoluminescence in irradiated GaN. Accordingly, another object of the current study iss to obtain experimental confirmation of this prediction. A brief report on preliminary results of experimental and theoretical studies of enhancement of surface amorphous layer formation was published earlier in Ref. 31.

In this paper, irradiation-induced effects were studied experimentally and by MD simulation of multiple subsequent impacts up to fluences matching those used in the experiments. We measured structural damage formation and photoluminescence decay in $\mathrm{GaN}$ bombarded with atomic ( $\mathrm{F}$ and $\mathrm{P}$ ) and molecular $\left(\mathrm{PF}_{2}\right.$ and $\left.\mathrm{PF}_{4}\right)$ ions with an energy $0.6 \mathrm{keV} / \mathrm{amu}$ at room temperature. Additionally, we performed irradiation by $\mathrm{Ag}$ (107.9 amu) ions, to analyze the mass effect on PL and damage production, since $\mathrm{Ag}$ has the atomic mass close to the mass of the molecular ion $\mathrm{PF}_{4}$ 
Preprint of paper published as Journal of Physics. D, Applied Physics. 50 (2017) 505110.

(106.9 amu). The combination of theoretical calculations and analysis of experimental data gives us the possibility to clarify the basic aspects of formation of different defect types under different irradiation conditions on one hand, and the relevance of different defect types to optical properties of $\mathrm{GaN}$, on the other hand.

\section{Methods}

\subsection{Experimental}

Silicon doped wurtzite (0001) GaN epilayers, grown by metal-organic vapor phase epitaxy at the Ioffe Institute (St. Petersburg, Russian Federation), $2 \mu \mathrm{m}$ thick, on $c$-plane sapphire substrates, were implanted at room temperature with atomic $\mathrm{F}, \mathrm{P}, \mathrm{Ag}$ and molecular $\mathrm{PF}_{2}, \mathrm{PF}_{4}$ ions with an energy $0.6 \mathrm{keV} / \mathrm{amu}$. The choice of cluster ions (i.e. $\mathrm{PF}_{\mathrm{n}}$ with $n=2$ and 4), instead of mono-elemental clusters $A_{n}$ was due to experimental constraints of the ion accelerator used in this work. Ag was specifically chosen due to its comparable mass with $\mathrm{PF}_{4}$. Implantation was carried out at room temperature at $7^{\circ}$ off the [0001] direction to minimize channeling effects. As discussed in detail in [17,34], irradiation-induced effects can be conveniently studied if the following parameters are kept constant: ion energy normalized to amu, ion fluence in terms of the number of displacements per target atom (DPA), and ion beam flux in terms of DPA s${ }^{-1}$. In this case, the same volume-averaged amount of displacements can be expected in the target according to the binary collision approximation, and hence the same damage level should appear in the target in all cases if the damage is produced in linear collision cascades. All the irradiation conditions used in this study (see Table 1) comply with these requirements. DPA values were calculated with the TRIM code [35] (version SRIM-2006.02) as DPA $=n_{v} \times \Phi / n_{\text {at }}$, where $\Phi$ is ion fluence in ions per $\mathrm{cm}^{2}, n_{v}$ is an average number of vacancies produced per unit of depth by one ion at the depth of maximum nuclear energy loss, and $n_{\mathrm{at}}=8.85 \cdot 10^{22} \mathrm{~cm}^{-3}$ is the GaN atomic concentration. Effective threshold energies for atomic displacements were $25 \mathrm{eV}$ for both $\mathrm{Ga}$ and $\mathrm{N}$ sub-lattices. The TRIM simulated $n_{v}$ for the ions considered are presented in Fig. 1. The estimation of the amount of vacancies produced by molecular $\mathrm{PF}_{\mathrm{n}}$ ions was made as a linear combination of vacancies generated by corresponding number of constituent atomic ions $\mathrm{DPA}_{\mathrm{PFn}}=\mathrm{DPA}_{\mathrm{P}}+n \times \mathrm{DPA}_{\mathrm{F}}$, where $n=2,4$. As it is seen in Fig. $1, \mathrm{P}+4 \times \mathrm{F}, 3.3 \times \mathrm{P}$ and $5.8 \times \mathrm{F}$ show similar vacancy concentration and distribution in the BCA approximation, which let us compare molecular and monatomic doses. We neglect electronic energy loss in the DPA definition, as it has negligible influence on structure damage formation in the keV ion energy regime. All experimental irradiation conditions used in these studies are summarized in Table 1. Ion beam fluxes used to study PL properties and damage formation were chosen in order to get reasonable irradiation times. The flux effect at such a low ion beam current will play a very small role, and we neglect it in our further analysis.

\begin{tabular}{|c|c|c|c|c|c|}
\hline & $\mathrm{F}$ & $\mathrm{P}$ & $\mathrm{PF}_{2}$ & $\mathrm{PF}_{4}$ & $\mathrm{Ag}$ \\
\hline Ion energy, $\mathrm{keV}$ & 11 & 19 & 42 & 65 & 65 \\
\hline $\mathrm{n}_{\mathrm{V} \_ \text {max }}, \AA^{-1} \operatorname{Ion}^{-1}$ & 0.85 & 1.46 & 3.13 & 4.83 & 4.51 \\
\hline $\mathrm{j}, \mathrm{nA} \mathrm{cm}^{-2}$ & 6.0 and 83 & 3.5 and 48 & 1.6 and 22 & 1.1 and 15 & 1.2 and 16 \\
\hline Flux, $10^{-4} \mathrm{DPA} / \mathrm{s}$ & 0.36 and 5 & 0.36 and 5 & 0.36 and 5 & 0.36 and 5 & 0.36 and 5 \\
\hline Fluence per 1 DPA, $10^{14} \mathrm{~cm}^{-2}$ & 10.3 & 6.00 & 2.80 & 1.82 & 1.95 \\
\hline $\begin{array}{l}\text { DPA in MD simulation cell } \\
\text { after } 1 \text { ion impact }\end{array}$ & $6.05 \times 10^{-5}$ & $1.04 \times 10^{-4}$ & $2.23 \times 10^{-4}$ & $3.44 \times 10^{-4}$ & $3.21 \times 10^{-4}$ \\
\hline
\end{tabular}

Table 1: Experimental irradiation conditions used in this study, and estimated DPA value corresponding to one impact of each projectile onto the calculation cell.

Implantation-produced disorder was measured by Rutherford backscattering spectrometry in channeling mode $(\mathrm{RBS} / \mathrm{C})$ by $0.7 \mathrm{MeV} \mathrm{He}^{++}$ions incident along the [0001] direction and 
Preprint of paper published as Journal of Physics. D, Applied Physics. 50 (2017) 505110.

backscattered to $103^{\circ}$. Both implantation and RBS/C analysis were performed using $500 \mathrm{keV}$ HVEE implanter.

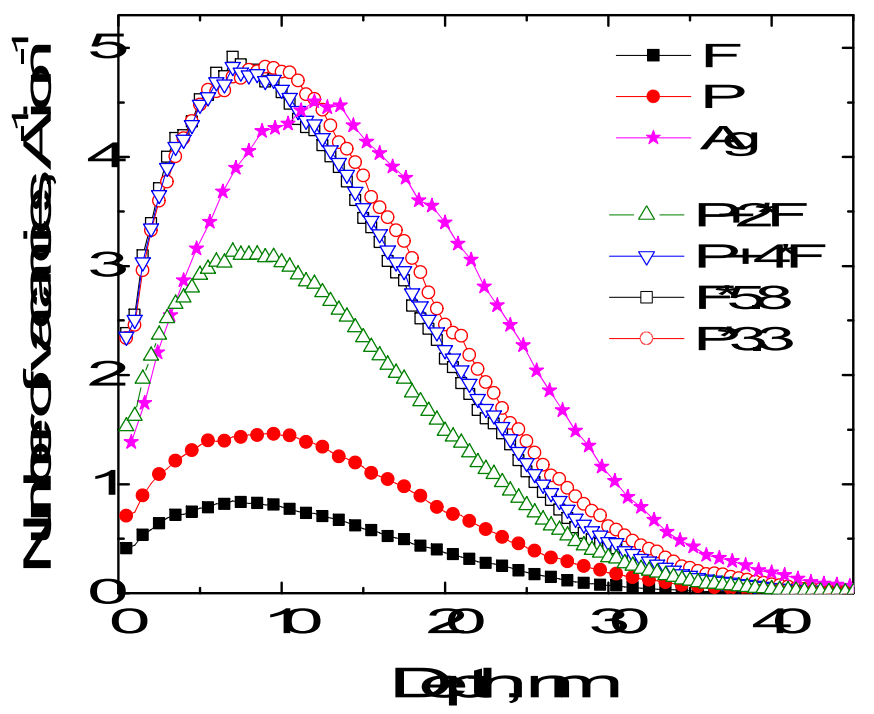

Figure 1: SRIM generated vacancy distributions (closed symbols) and scaled vacancy distributions (open squares and circles) for different monatomic projectiles together with linear combinations calculated for $\mathrm{PF}_{\mathrm{n}}$ molecules (open triangles). It is clearly seen that $\mathrm{PF}_{4}$ molecule generates 5.8 times more vacancies than $\mathrm{F}$ and 3.3 times more vacancies than P monatomic ions. Depth distributions are similar in all three cases (Color online)

Band edge recombination dynamics was studied by time-resolved photoluminescence using 2 ps optical pulses from a double cascade compressed Nd-YAG laser with a $340 \mathrm{~nm}$ central wavelength. The PL signal, spectrally integrated around the GaN band gap wavelength of 360 $\mathrm{nm}$, was measured at room temperature with a Hamamatsu C-979 streak camera. The temporal resolution of the system response was $8 \mathrm{ps}$. The decay time $t_{D}$ was determined as the time during which the PL signal reduced to $1 / \mathrm{e}$ of its maximum. This criterion provides the reference value $t_{D} \sim 270 \mathrm{ps}$ for as-grown samples. A more detailed experimental setup description can be found elsewhere [30].

\subsection{Molecular dynamics simulations}

The irradiation processes were simulated using the classical Molecular Dynamics (MD) method with the PARCAS code [36 - 38]. The wurtzite-structured GaN cells were constructed with an open surface in the [0001] direction. The interatomic potential used to describe the GaN substrate describes a wide range of structural properties including point defects in line with DFT calculations [39], and has been previously used successfully to study irradiation-induced defects $[33,40-42]$. The simulation cell size was $414 \times 386 \times 402 \mathrm{~A}^{3}$. The simulation cell containing a total of 5.5 million atoms was equilibrated at $300 \mathrm{~K}$ using Berendsen temperature and pressure control [43] before the irradiation runs. Details of the simulation procedure are given elsewhere $[33,40]$. Irradiation simulations were done by $\mathrm{F}, \mathrm{P}, \mathrm{PF}_{2}, \mathrm{PF}_{4}$ and $\mathrm{Ag}$ ions (see Table 1). The ion energy for each irradiation case was taken equal to the experimental value. The interactions in the $\mathrm{PF}_{\mathrm{n}}$ molecule was described by Lennard-Jones potentials, constructed to give the correct bond length and bond energies within the molecule [40].

The atomic or molecular ion was placed above the simulation cell surface, and directed towards it at the angle $7^{0}$ off the sample normal. Periodic boundary conditions were applied in the lateral directions $(x, y)$. The one-impact simulation time was $20 \mathrm{ps.} \mathrm{The} \mathrm{possible} \mathrm{influence}$ of the cell relaxation time on simulation results will be discussed below. After each impact, the 
Preprint of paper published as Journal of Physics. D, Applied Physics. 50 (2017) 505110.

damaged simulation cell was randomly shifted in the $x$ and y directions, after which periodic boundary conditions were applied again. In this manner, fifty cumulative irradiations were done by each projectile type. Three independent cumulative calculation sequences were completed to improve statistics.

After the irradiation simulation, the damage was analyzed using Voronoy polyhedra centered on each initial atom position. Polyhedra with no atoms were labeled as vacancies andpolyhedra with 2 or more atoms as interstitials. In addition, we also analyzed the formation of defect clusters and the cluster size distribution for all investigated cases. Cluster analysis was done by calculating the distance from each point defect to all other defects. All defects that were within a fixed cut off radius from each other were interpreted to be a part of the same defect cluster. The cutoff radius was chosen to be one and half a lattice constant (3.116 $\AA$ ).

We note that the distributions of the number of vacancies obtained in MD irradiated simulation cells corresponds closely to the distribution of vacancies for the same dose in DPA, calculated from SRIM [35]. For the comparison with experiment, we estimated the dose of irradiation in DPA in the similar manner as described in Sect. 2.1. In other words, number of cumulative impacts for different projectiles was chosen in such a way that atoms in simulated target experienced similar number of displacements (DPA). A single ion impact on a simulated surface corresponds to a fluence of $0.626 \times 10^{11}$ ions $/ \mathrm{cm}^{2}$. The corresponding doses in DPA for each type of an ion are shown in Table 1.

\section{Results and Discussion}

\subsection{Low dose regime. Photoluminescence decay study.}

As discussed above, energetic ion stopping in $\mathrm{GaN}$ target forms various defects, which in turn affect all properties of the material. One of possible techniques to investigate damage formation in $\mathrm{GaN}$ at the early irradiation stages, when only a few defects present in the crystal, is time-resolved photoluminescence (PL) studies. Structural defects act as a nonradiative sinks for photoexcited carriers, and the change observed in carrier dynamics will indicate the amount of damage formed. Fig. 2 (a) shows the measured decay of the PL signal spectrally integrated over $360 \mathrm{~nm}$ peak (see Refs. 30, 31) for GaN targets irradiated by monatomic and molecular ions to the dose of $0.8 \times 10^{-3} \mathrm{DPA}$. For clarity in the figure, only one of every five experimental points is shown by markers. PL decay in the unirradiated samples is also shown. Consistently to previous results $[8,31,44]$, PL decay in all samples can be treated as following a biexponential law. This is well known behavior of luminescence driven by nonradiative recombination on saturating sinks [44]. We determine characteristic decay time $t_{D}$ as the time required for the reduction of a PL signal by a factor of $e$. It is clearly seen from Fig. 2 (a) that molecular ion irradiation is more efficient in shortening PL decay time compare to its atomic constituents. Heavy Ag monatomic ion bombardment affects $t_{D}$ even stronger. This result is further illustrated in Fig. 2 (b), where the dose dependencies of decay time are presented. It is seen from Fig. 2 (b) that heavy atomic ion irradiation causes much faster PL decay time decrease than the molecular ions, which in turn is more effective than light atomic ions. Interestingly, the difference in $t_{D}$ for light atom $(\mathrm{F}, \mathrm{P})$ and molecule $\left(\mathrm{PF}_{4}\right)$ irradiations vanishes with ion dose. All three species give the same value of $\sim 40 \mathrm{ps}$ in the samples irradiated to $8 \times 10^{-}$ ${ }^{2}$ DPA. Taking into account the very low implantation fluences, the reduction of the $t_{D}$ in the implanted samples should be attributed to damage rather than to chemical effects of the implanted ions. Hence the difference in PL decay time after different ion irradiation is a signature of different types of different concentration of defects formed in the $\mathrm{GaN}$ by different ions. Also in [33], based on a single impact MD simulations it was suggested that the defect clusters can be one of the important reasons for fast PL decay. Below we affirm that point with the cumulative simulation results. 

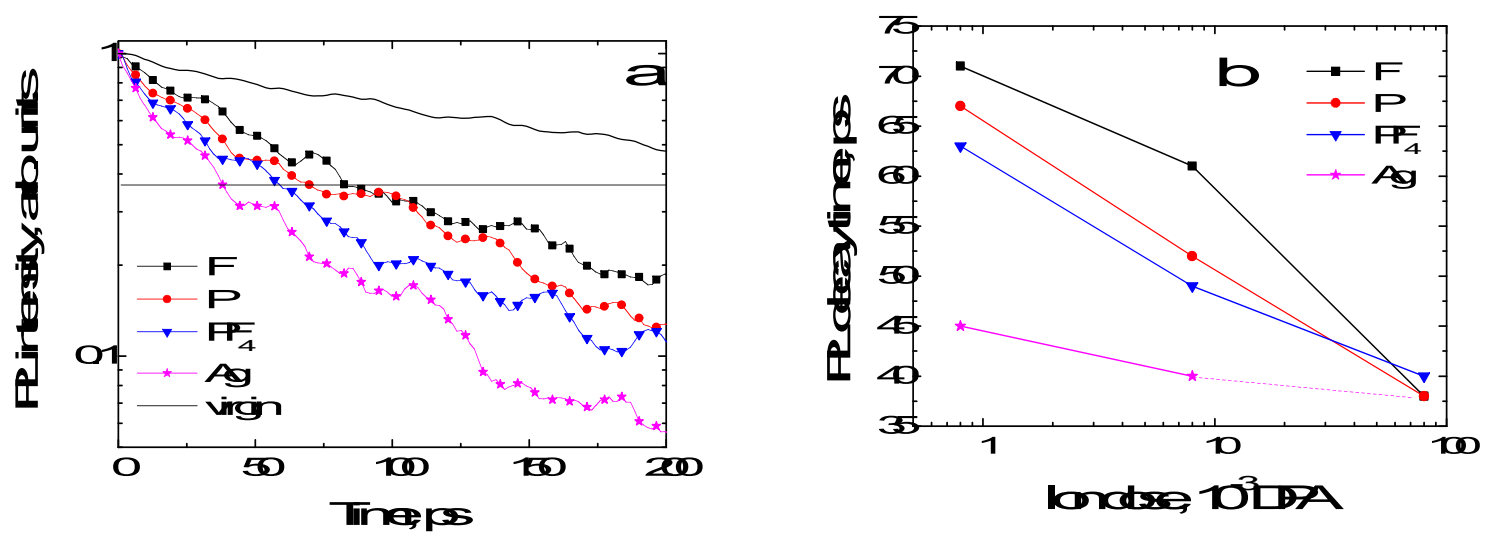

Figure 2. Time dependencies of photoluminescence intensity from virgin $\mathrm{GaN}$ and targets irradiated with different projectiles to a dose of $0.8 \times 10^{-3}$ DPA (a) and corresponding PL decay times as a function of ion dose (b). (Color online)

\subsection{High dose regime. RBS/C study of structure damage formation}

To perform an investigation of structure damage formed in a sample by RBS/C technique, one needs to achieve at least $\sim 3 \%$ of relative disorder, which requires relatively high ion doses. It is obvious that such a high amount of lattice damage and, consequently, number of nonradiative recombination levels formed, completely suppresses radiative recombination in the sample. Fig. 3 presents typical RBS/C spectra obtained from GaN samples irradiated with different ions up to the same dose of 3 DPA. Irradiation to other doses from 1.5 to 10 DPA gives RBS/C spectra with very similar behavior to shown in Fig. 3. In all cases studied, the bi-modal lattice damage formation is found consistent with previous reports [4, 7, 9, 13-17, and 31]. One defect peak appears in the bulk and another one, corresponding to a thin amorphous (or nanocrystalline) layer $[4,45,46]$, forms at the sample surface. However, spectra obtained after different projectile implantations differ from each other, even though an equal number of identically distributed point defects were produced in the target in all cases, according to the binary collision approximation.

Positions of bulk peak maxima for all projectiles are deeper than the maxima of elastic energy loss predicted by binary collision approximation (indicated by arrows in Fig. 3). This clearly shows the high importance of the secondary defect formation processes in radiationinduced stable damage accumulation in $\mathrm{GaN}$, as suggested in [47]. Fig. 3 shows that light $\mathrm{F}$ and $\mathrm{P}$ ions as well as $\mathrm{PF}_{\mathrm{n}}$ molecules give rise to an almost similar bulk defect peak. Slightly higher values seen in the molecular spectra (Fig. 3) are due to higher de-channeling value of the probing He ions in the thick disordered layer, which appeared in these cases at the target surface. The integrated peak areas are almost the same. The bulk peak produced by heavy Ag ions is located deeper in the sample, and its area is bigger than that for other ions. This behavior could be understood taking into account two reasons: (i) slightly deeper position of the maximum of nuclear energy loss (as indicated in Fig.1), and (ii) correlation of the bulk damage buildup with the density of collision cascades. The heavy Ag ion has high nuclear energy loss and will form dense collision cascades at all penetration depths which gives high bulk damage value. In the case of molecular $\mathrm{PF}_{\mathrm{n}}$ ion bombardment, overall collision cascade density is determined by the individual cascades created by $\mathrm{P}$ and $\mathrm{F}$ components. The atoms comprising molecule will spread fast with depth [40] and consequently, we should not find significant difference in the densities of cascades formed by molecular $\mathrm{PF}_{\mathrm{n}}$ or its constituent monatomic ions in the crystal bulk. Hence, molecule and its constituents will form similar damage. 


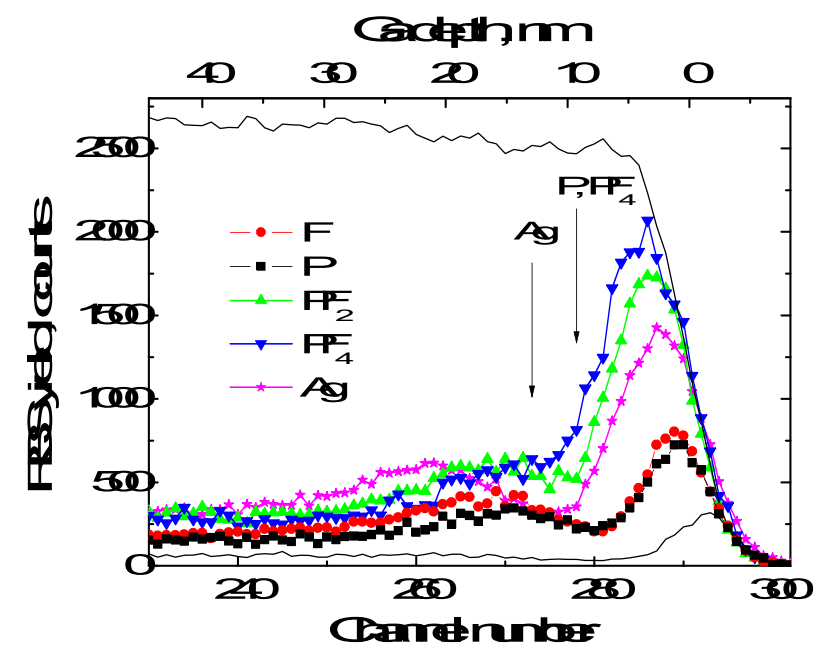

Figure 3. RBS/C spectra for GaN irradiated to the dose of 3 DPA by different ions as indicated in the legend. The positions of the maxima of vacancy distributions generated by different ions as predicted by TRIM (see Fig. 1) are shown by arrows. Random and virgin spectra are shown by black lines. (Color online).

It is also seen from Fig. 3 that the surface damage peak appears after each of the ions used. This damage peak corresponds to thin surface amorphous layer (SAL) formed at the GaN sample surface [4]. The SAL formed by molecular ion irradiation is thicker compared to that formed by irradiation with constituent monatomic species, i.e. a molecular effect (ME) takes place. ME efficiency increases with the size of molecules. Indeed, $\mathrm{PF}_{4}$ ions produce thicker amorphous layer than $\mathrm{PF}_{2}$ ions. Heavy $\mathrm{Ag}$ ion irradiation produces a thicker SAL than that of light $\mathrm{F}$ and $\mathrm{P}$ ions, but thinner than the one produced by molecules. SAL formation indicates that the surface is a nucleation site for point defects. It was earlier shown that the lattice amorphization rate at the surface linearly increases with the amount of point defects coming to the amorphous/crystalline interface [47, 48]. Overlapping of individual collision cascades formed by atoms comprising the $\mathrm{PF}_{\mathrm{n}}$ molecule locally increases cascade density at the sample surface and, in turn, increases the number of point defects produced by molecular ions in the surface vicinity. Some of these mobile defects will come to amorphous/crystalline interface, causing faster SAL growth. Analysis of experimental data suggests that silver ions generate more point defects than fluorine or phosphorus, but they are not as efficient at the sample surface as the $\mathrm{PF}_{\mathrm{n}}$ molecules.

Thus, superlinear collision cascade formation plays an important role in ion-beam-induced damage formation both in the GaN bulk and at the surface. However, the relative role of different cascade-related mechanisms responsible for the effects observed cannot be derived from the above data and require additional studies. It is important to understand the process at the atomistic level. In the Sect. 3.3 we present results of cumulative MD simulations of radiation damage formation during irradiation of $\mathrm{GaN}$ with all mentioned ion species.

\subsection{Cumulative MD simulation}

We performed cumulative molecular dynamic simulations for direct modeling of processes developing in $\mathrm{GaN}$ target during stopping of the ions.

Figure 4 (a) shows accumulation of the total amount of vacancies produced by different projectiles with cumulatively increasing number of impacts. The distributions and amount of interstitials follow the same trend and hence are not presented here. The quantity of point defects increases almost linearly for all projectiles. Scaling to a similar number of displacements (DPA) evidences that at low dose range up to $10^{-2} \mathrm{DPA}$, all projectiles except Ag produce a comparable number of point defects (Fig. 4 (b)). A slight deviation from linear dependence is seen for heavy 
Preprint of paper published as Journal of Physics. D, Applied Physics. 50 (2017) 505110.

$\mathrm{Ag}$ and $\mathrm{PF}_{4}$ ions at high doses. Overall, we found a higher number of defects produced by heavy $\mathrm{Ag}$, and almost no difference in the total amount of point defects generated by molecular and light atomic ions even close to the sample surface, where it was expected. This result is somewhat intriguing, but close to our single impact simulation data [33].
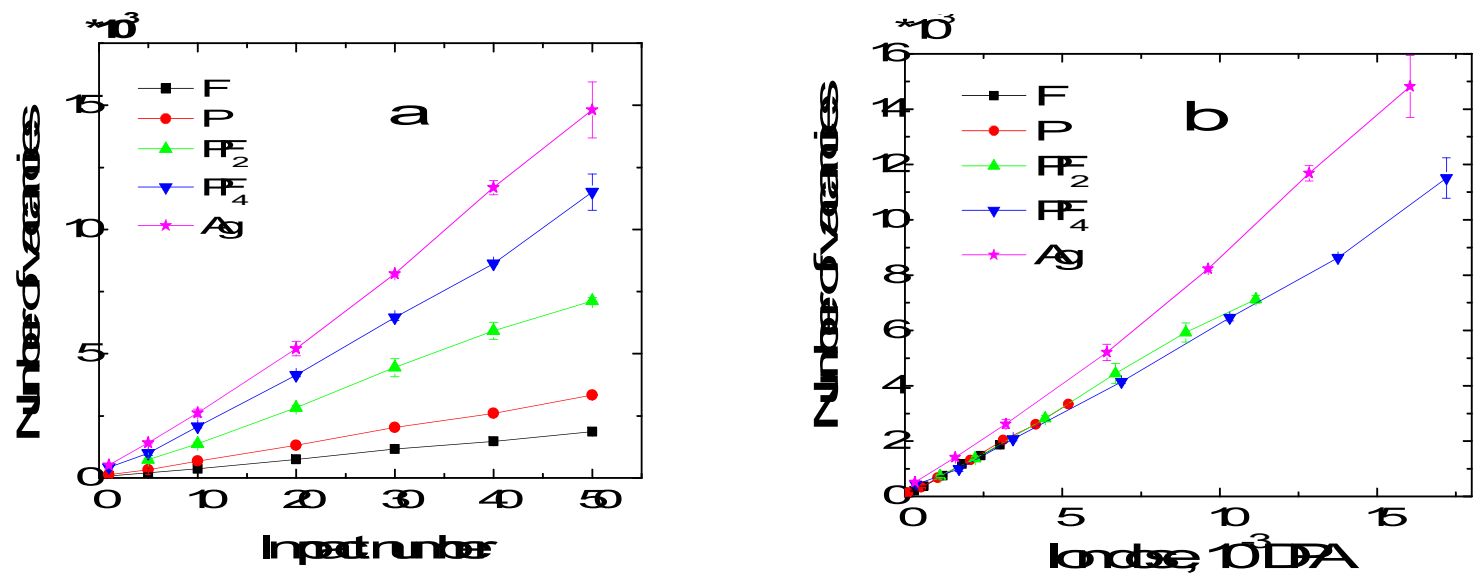

Figure 4: (a) Vacancy accumulation for GaN substrate irradiated by different projectiles (as indicated in the legend) with increasing number of cumulative impacts. (b) Number of defects as a function of dose expressed in DPA.

Figure 5 shows the depth distribution of point defects (vacancies) simulated for all projectiles. To compare defect production by all different projectiles, a different number of ion impacts were used to reach the same dose of $3 \times 10^{-3} \mathrm{DPA}$, which for $\mathrm{F}, \mathrm{P}, \mathrm{PF}_{2}, \mathrm{PF}_{4}$ and $\mathrm{Ag}$ were 50, 30, 15, 9 and 9 ion impacts, respectively. The results are presented in Fig.5. No distinguishable difference is seen between depth distributions of point defects produced by single $(\mathrm{P})$ and molecular $\left(\mathrm{PF}_{4}\right.$ and $\left.\mathrm{PF}_{2}\right)$ ions. Simulation of $\mathrm{Ag}$ ion impacts shows a slightly higher number of simple point defects, whereas light $\mathrm{F}$ ions give a bit less point defects. The depth of the maxima of the vacancy distributions is around $10 \mathrm{~nm}$, which coincides well with TRIM simulated position of maximum of nuclear energy loss. Previously, on the base of single MD simulated impact data, we have reported that the $\mathrm{PF}_{4}$ molecule produces an amount of point defects which is comparable to a linear combination of defects produced by monatomic ions $\mathrm{P}+4 \times \mathrm{F}[33]$. Here in cumulative simulations, we see the same behavior of a molecule and its constituents. 


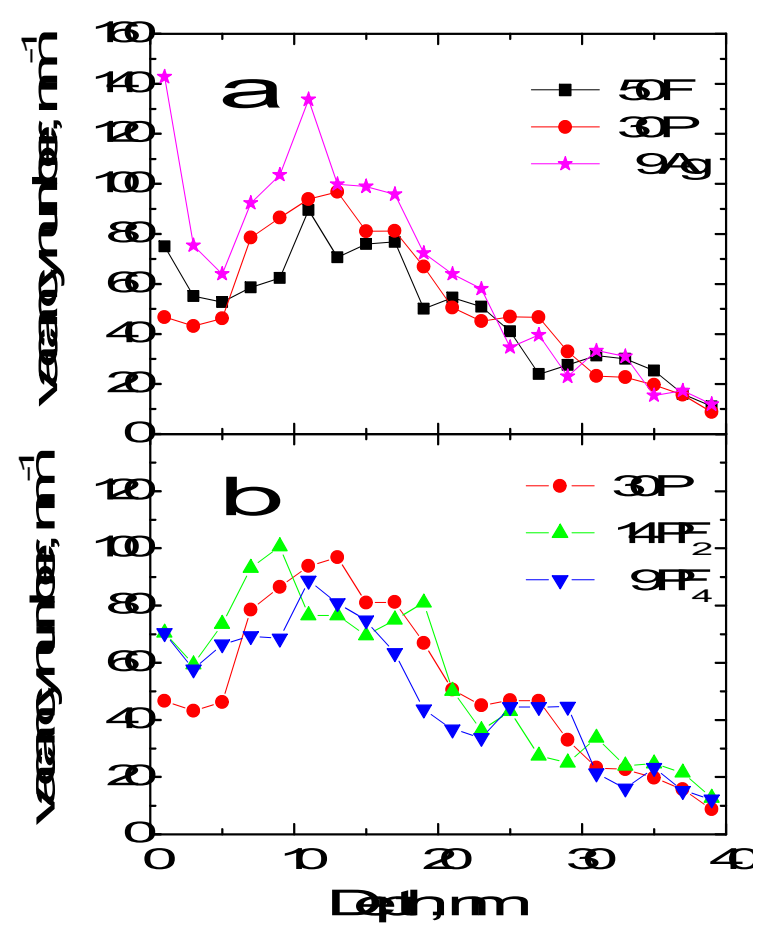

Figure 5. MD simulated depth distribution of point defects produced by different projectiles after cumulative impacts to a dose of $3 \times 10^{-3}$ DPA. Impact numbers are indicated. (Color online)

As we saw earlier [33], such an analysis of single point defect creation is not sufficient for $\mathrm{GaN}$ structures. A difference in damage formation under atomic and molecular ion irradiation can appear in the production of defect clusters. Fig. 6 presents depth distributions of defect clusters consisting of vacancies and interstitials produced by different projectiles after cumulative simulation to the dose of $5.2 \times 10^{-3} \mathrm{DPA}$. This value corresponds to 50,15 and 16 ions for $\mathrm{P}_{2} \mathrm{PF}_{4}$ and $\mathrm{Ag}$ ions respectively. The numbers of small (2-20 defects) and large ( $>20$ defects) clusters per unit depth are shown in figures 6 (a) and 6 (b), respectively. In Fig. 6(a) no significant difference in small cluster formation is seen; indeed, light $\mathrm{P}$, heavy Ag and molecular $\mathrm{PF}_{4}$ ions produce almost same small cluster distributions. However, in the Fig. 6(b) it is seen that in the vicinity of the surface, molecules produce more large clusters than atomic $\mathrm{P}$ ions. Ag produces a bigger number of large defect clusters than the other ions over all penetration depths. This effect is further illustrated by Fig. 7, where the average number of the large defect clusters obtained within $5 \mathrm{~nm}$ thick slices of the simulation cells at two different depths: near the surface and at the depth of the maximum nuclear stopping powers for all compared ions is presented as a function of ion dose. Fig. 7 (a) shows clusters at the first $5 \mathrm{~nm}$ from the surface and Fig. 7(b) presents those around maximum of nuclear energy loss (5.5 to $10.5 \mathrm{~nm}$ for $\mathrm{P}, \mathrm{PF}_{2}, \mathrm{PF}_{4}$ and 6.5 to $11.5 \mathrm{~nm}$ for Ag ions). Lines are results of linear fitting. It is seen that large defect clusters also accumulate linearly with fluence. The accumulation rate (the curve slope) at the surface (see Fig. 7(a) and Table 2) is $190 \mathrm{DPA}^{-1} \mathrm{~nm}^{-1}$ for $\mathrm{PF}_{4}$ ions and about $90 \mathrm{DPA}^{-1} \mathrm{~nm}^{-1}$ for $\mathrm{P}$ and $\mathrm{PF}_{2}$, which is a factor of two less. In the sample bulk, we found almost similar cluster production efficiency for all three $\mathrm{P}, \mathrm{PF}_{2}$ and $\mathrm{PF}_{4}$ ions (see Fig. 7(b)). Heavy Ag ions in both cases generate clusters with the highest rate. 
Preprint of paper published as Journal of Physics. D, Applied Physics. 50 (2017) 505110.

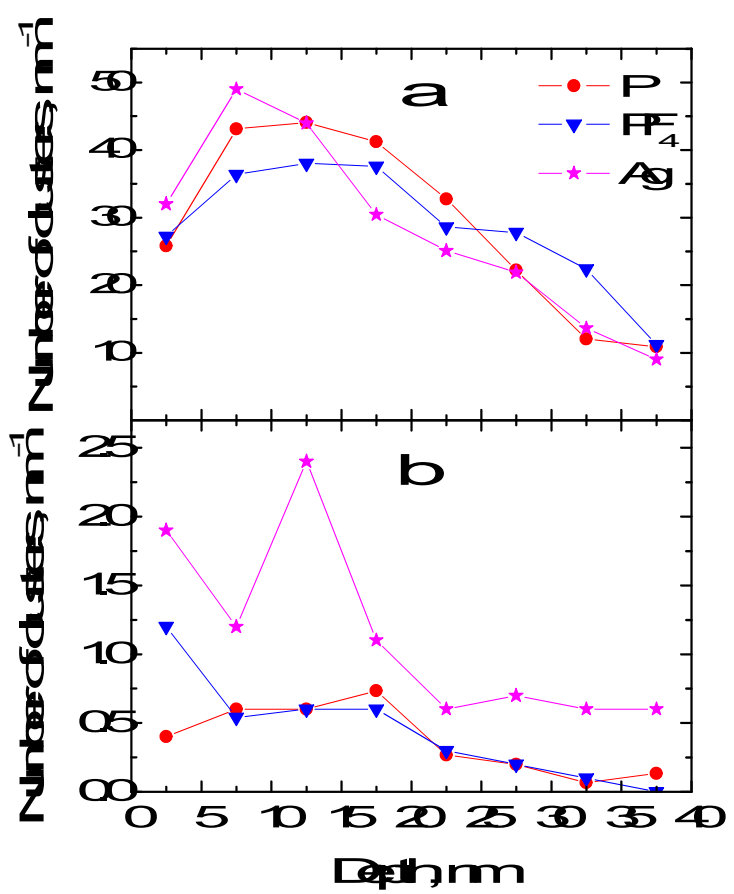

Figure 6: Depth distribution of small (2-20 defects, a) and large ( $>20$ defects, b) defect clusters produced by $\mathrm{Ag}, \mathrm{PF}_{4}$ and $\mathrm{P}$ ions after cumulative irradiations corresponding to a similar dose of $5.2 \times 10^{-3}$ DPA.

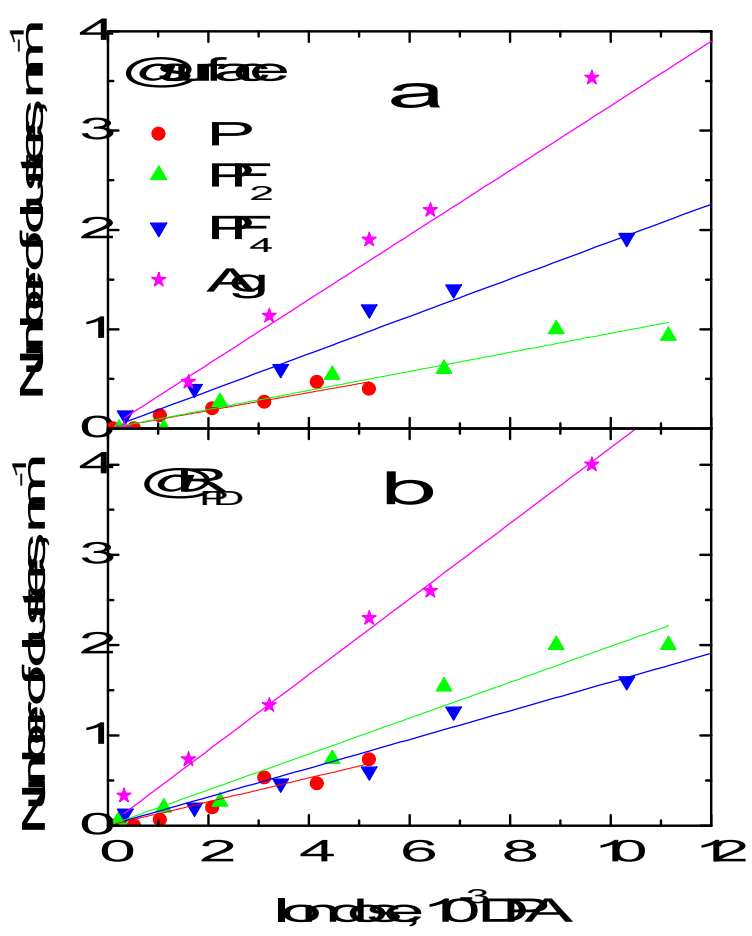

Figure 7: Average number of large $(>20)$ defect clusters produced by different projectiles at the sample surface (a) and around the maximum of nuclear energy loss (b) as a function of ion dose. 
Preprint of paper published as Journal of Physics. D, Applied Physics. 50 (2017) 505110.

\begin{tabular}{|l|l|l|}
\hline Ion & $\begin{array}{l}\text { Slope @ surface } \\
\left(\mathrm{DPA}^{-1} \mathrm{~nm}^{-1}\right)\end{array}$ & $\begin{array}{l}\text { Slope @ R } \\
\left(\mathrm{DPA}^{-1} \mathrm{~nm}^{-1}\right)\end{array}$ \\
\hline $\mathrm{P}$ & $90 \pm 14$ & $132 \pm 20$ \\
\hline $\mathrm{PF}_{2}$ & $96 \pm 12$ & $198 \pm 22$ \\
\hline $\mathrm{PF}_{4}$ & $188 \pm 8$ & $159 \pm 10$ \\
\hline $\mathrm{Ag}$ & $324 \pm 20$ & $419 \pm 46$ \\
\hline
\end{tabular}

Table 2. Large ( $>20$ point defects) defect cluster accumulation rate for different projectiles at the sample surface and aroundthe maximum of nuclear energy loss.

\subsection{Discussion}

We note that the same DPA corresponds to the same number of generated vacancies as defined by the binary collision approximation.

Both the experimental analysis of ion-induced structural damage formation, as well as the time-resolved PL measurements, indicates that formation of denser collision cascade results in more efficient damage production. The PL decay time decreases following the order of applied ions as $\mathrm{F}, \mathrm{P}, \mathrm{PF}_{2}, \mathrm{PF}_{4}$, and $\mathrm{Ag}$ (see Figs. 2 and 3). The efficiency of bulk damage accumulation grows with ion mass. Heavy $\mathrm{Ag}$ and especially $\mathrm{PF}_{4}$ ions form thicker SAL than others. The cumulative MD simulations give us insight to the atomistic level of processes which take place in $\mathrm{GaN}$ during the stopping of an ion.

First of all, two cascade-density-related phenomena were found in the cumulative MD results for heavy Ag ion implantation: (i) nonlinear increase of in-cascade point defect generation and (ii) enhanced big defect cluster formation. Indeed, the cascade density is very high in this case, and the atom interaction scenario changes from simple two-body collisions (linear collision cascades) treated by the binary collision approximation. Hence, an increased number of single point defects appears. Moreover, all the atoms in some small volumes can be set into motion, giving rise to disordered regions in the form of defect clusters. Both these effects occur throughout the entire range of silver ions. Although Ag and $\mathrm{PF}_{4}$ have comparable mass, the atoms comprising a molecule separate in the first surface layers, as it was shown in $[40,49]$. That is why in the bulk we do not observe any significant difference in point defect production between a molecule and its constituents. However, enhanced big defect cluster formation in the thin subsurface layer was found during $\mathrm{PF}_{4}$ irradiation compared to monatomic ions (Fig. 7(a)). It reveals the cascade density effect in damage formation during molecular ion implantation. It is reasonable to assume [33] that such defect clusters will be stable and operate as damage nucleation sites, which, in turn, will also enhance stable damage accumulation. On the other hand, we found no difference in defect cluster formation by $\mathrm{PF}_{2}$ and $\mathrm{P}$ ions (Fig. 7). Moreover, silver generates clusters more efficiently than $\mathrm{PF}_{4}$, but it is less efficient in SAL formation compared to both molecular ions (Fig. 2). This fact suggests the important role of point defect diffusion and post-cascade interaction processes. These processes are not described by MD simulations, because of the very long times needed to simulate the long-term-diffusiondriven processes. The inevitable difference between experiment and simulations is the magnitude of the ion flux, which was five orders of magnitude higher in the MD simulations. Indeed, in the experiments, the average time between sequential arrivals of ions at the surface area of the simulation cell is about microseconds, which is enough for mobile point defects to run out or recombine. The simulation time ( $t=20 \mathrm{ps}$ between ion impacts) would not allow such processes to be completed. This means the local point defect concentration will be overestimated, and the relative cluster formation probability will also significantly increase in the experimental situation. The evidence of the ME vanishing at high ion beam current was reported in [21]. Hence, the experimentally observed cascade density effects can also appear due to nonlinear secondary defect formation in $\mathrm{PF}_{\mathrm{n}}$ cascades. 
Considering that the experimentally found similar PL decay time for all ions at high dose, we note that effective nonequilibrium charge carrier excitation takes place up to 2 extinction lengths deep in the GaN crystal. This depth is about $120 \mathrm{~nm}$ [50], which is notably deeper than the damaged layer depth $(\sim 30 \mathrm{~nm})$, where nonradiative recombination centers are formed by the ion beam. Of course, some more damage will appear deeper in the bulk due to point defect diffusion [45, 46], but this fact will not significantly affect the following argumentation. At a high enough dose we obtain relatively thin $(<30 \mathrm{~nm})$ subsurface layer with no luminescence and still undamaged crystal bulk deeper in the sample. Charge carrier diffusion from that area to the damaged layer can be treated as nonradiative "surface recombination" at the damaged/undamaged region interface. It is obvious that the photo-excited charge carrier distribution will become almost the same for all ions at high enough "surface recombination" rate, i.e. at a high enough damage level. Hence, the PL decay time might tend to some identical value independently of the way of damage production. This effect is seen in the experimental data in Fig. 2 (b). Indeed, according to the MD data, lattice disorder generated by all kinds of ions in that subsurface region becomes almost identical at high enough dose (see Fig. 7). At the same time, the dose needed to reach that state depends on ion type, which causes initial difference in PL decay times.

\section{Conclusions}

In conclusion, irradiation-induced formation of defects in $\mathrm{GaN}$ under monatomic and molecular ion bombardment was experimentally investigated in low and high dose regime by means of studying of PL decay time shortening and structure damage. Molecular ion irradiation was found to produce thicker surface amorphous layers and to be more efficient in shortening the PL decay time in comparison with its constituent atomic species. Heavy Ag ion was even more efficient in PL quenching and bulk damage formation than both light atoms and molecules. Classical MD simulations of cumulative sequential ion impacts were used to elucidate the defect production efficiency of light, heavy and molecular ion irradiation in GaN. The calculated results show a linear increase in point defect production with increasing number of cumulative impacts for all projectiles. No significant difference was found in MD predicted simplest point defect formation by molecular ions $\left(\mathrm{PF}_{2}, \mathrm{PF}_{4}\right)$ and their constituent monatomic ions $(\mathrm{F}, \mathrm{P})$. Enhanced large defect cluster formation in the thin subsurface layer during $\mathrm{PF}_{4}$ irradiation compared to monatomic ions was found in MD simulations, and explained by locally increased collision cascade density. Heavy Ag ions show high efficiency in both point and cluster defect production due to formation of dense collision cascades over all penetration depth. These large damage clusters can thus be associated with the shortening of the PL decay time and play an important role in structure damage formation in ion-beam-irradiated $\mathrm{GaN}$.

\section{Acknowledgement}

Authors are grateful to Dr. Wsevolod Lundin from Ioffe Institute, St. Petersburg, Russia for providing the GaN samples used in this study. Work was supported by Academy of Finland under the ENIGAZ project, and by Russian RFBR. Grants of computer time from the Center for Scientific Computing in Espoo, Finland, are gratefully acknowledged. 
Preprint of paper published as Journal of Physics. D, Applied Physics. 50 (2017) 505110.

\section{References}

1. H. P. Maruska and J. J. Tietjen, Appl. Phys. Lett. 15327 (1969).

2. M. S. Shur, R. Gaska, and A. Bykhovski, Solid-State Electronics 431451 (1999).

3. S. J. Pearton, J. C. Zolper, R. J. Shul, F. Ren, J. Appl. Phys. 861 (1999)

4. S. O. Kucheyev, J. S. Williams, and S. J. Pearton, Mater. Sci. Eng.R. 3351 (2001).

5. S. J. Pearton, B. S. Kang, S. Kim, F. Ren, B. P. Gila, C. R. Abernathy, J. Lin, and S. N. G.

Chu, J. Phys.: Condens. Matter 16 R961 (2004).

6. H. Morkoç, S. Strite, G. B. Gao, M. E. Lin, B. Sverdlov and M. Burns, J. Appl. Phys. 76, 1363 (1994).

7. S. O. Kucheyev, H. Boudinov, J. S. Williams, C. Jagadish, and G. Li, J. Appl. Phys. 91, 4117 (2002).

8. A. Pinos, S. Marcinkevicius, M. Usman, A. Hallen. Appl. Phys. Lett., 95112108 (2009).

9. M.Nastasi, J.W.Mayer, Ion implantation and Synthesis of Materials, Chapt. 7, (Springer-

Verlag Berlin Heidelberg, 2006).

10. R. A. Brown and J. S. Williams, Phys. Rev. B 5512852 (1997).

11. S. O. Kucheyev, J. S. Williams, C. Jagadish, J. Zou, G.Li, Phys. Rev. B, 627510 (2000).

12. B. Pipeleers, S. M. Hogg, and A. Vantomme, J. Appl. Phys. 98, 123504 (2005).

13. F. Gloux, T. Wojtowicz, P. Ruterana, K. Lorenz, E. Alves, J. Appl. Phys. 100, 073520

(2006).

14. M. Ishimaru, Y. Zhang, and W. J. Weber, J. Appl. Phys. 106, 053513 (2009).

15. E. Wendler, A. Kamarou, E. Alves, O. K. Gartner, W. Wesch, Nucl. Instr. and Meth. B 206, 1028 (2003).

16. E. Wendler, Nucl. Instr. and Meth. B 267, 2680 (2009).

17. S. O. Kucheyev, A. Y. Azarov, A. I. Titov, P. Karaseov, and T. M. Kuchumova, J. Phys. D: Appl. Phys. 42085309 (2009).

18. J. Gyulai, T. Lohner, M. Fried, N. Q. Khanh, Gen Qing Yang, and Z. Toth, Radiat. Eff. 140, 49 (1996).

19. Jian-Yue Jina, Jiarui Liu, Paul A. W., van der Heide, Wei-Kan Chu, Appl. Phys. Let. 76, $574(2000)$.

20. A. I. Titov, S. O. Kucheyev, V. S. Belyakov, and A. Y. Azarov, J. Appl. Phys. 903867 (2001).

21. A. I. Titov, A. Y. Azarov, L.M.Nikulina, S.O.Kucheyev, Phys. Rev. B 73064111 (2006).

22. A.I. Titov, A.Yu. Azarov, L.M. Nikulina, S.O. Kucheyev, Nucl. Instr. Meth. Phys. Res. B 256207 (2007).

23. B. Sundaravel, C. David, A. K. Balamurugan, S. Rajagopalan, A. K. Tyagi, B. K. Panigrahi, K. G. M. Nair, and B. Viswanathan, Phys.Rev. A 73, 042902 (2006).

24. H. P. Lenka, B. Joseph, P. K. Kuiri, G. Sahu, P. Mishra, D. Ghose and D. P. Mahapatra, J. Phys. D: Appl. Phys. 41, 215305 (2008).

25. V.N. Popok, Materials Science and Engineering R 72, 137 (2011).

26. C. David, C. Varghese Anto, M. Dholakia, S. Chandra, K. G. M. Nair, B. K. Panigrahi, P. S. Raman, S. Amirthapandian, G. Amarendra, and J. Kennedy, J. Appl. Phys., 117, 014312 (2015). 27. D. A. Thompson, Radiat. Eff. 56, 105 (1981).

28. J.A. Davies, in: J.S. Williams, J.M. Poate (Eds.), Ion Implantation and Beam Processing (Academic Press, Sydney, 1984).

29. S. O. Kucheyev, J. S. Williams, A. I. Titov, G. Li, and C. Jagadish, Appl. Phys. Lett. 78 2694 (2001).

30. P. A. Karaseov, K. V. Karabeshkin, A. I. Titov, V. B. Shilov, G. M. Ermolaeva, V. G. Maslov, and A. O. Orlova, Semiconductors. 48446 (2014).

31. P. A. Karaseov, K.V Karabeshkin, E.E.Mongo, A. I. Titov, M. W. Ullah, A. Kuronen, F. Djurabekova, K. Nordlund, Vacuum 129166 (2016).

32. C. Björkas, K. Nordlund, K. Arstila, J. Keinonen, V. D. S. Dhaka, and M. Pessa. J. Appl. Phys., 100053516 (2006) 
Preprint of paper published as Journal of Physics. D, Applied Physics. 50 (2017) 505110.

33. M. W. Ullah, A. Kuronen, K. Nordlund, F. Djurabekova, P. Karaseov, K. V. Karabeshkin, and A. I. Titov, J. Appl. Phys. 114183511 (2013).

34. A.Yu. Azarov, S.O. Kucheyev, A.I. Titov, P.A. Karaseov, J. Appl. Phys., 102083547 (2007)

35. J.F.Ziegler, J.P. Biersack, U.Littmark,: The Stopping and Range of Ions in Solids.

Pergamon Press, New York (1985); J. F. Ziegler, SRIM-2013 software package, available online at http://www.srim.org.

36. K. Nordlund, M. Ghaly, R. S. Averback, M. Caturla, T. Diaz de la Rubia, and J. Tarus, Phys. Rev. B 577556 (1998).

37. M. Ghaly, K. Nordlund, and R. S. Averback, Phil. Mag. A 79795 (1999).

38. K. Nordlund, Comput. Mater. Sci. 3448 (1995).

39. J. Nord, K. Albe, P. Erhart, and K. Nordlund, J. Phys.: Condens. Matter 155649 (2003).

40. M. W. Ullah, A. Kuronen, K. Nordlund, F. Djurabekova, P. Karaseov, and A. I. Titov, J.

Appl. Phys. 112043517 (2012).

41. M. W. Ullah, A. Kuronen, K. Nordlund, F. Djurabekova, A. I. Titov, and P. Karaseov.

Vacuum, 10588 (2013)

42. M.W. Ullah, A. Kuronen, A. Stukowski, F. Djurabekova, and K. Nordlund. J. Appl. Phys., 116, 124313 (2014)

43. H. J. C. Berendsen, J. P. M. Postma, W. F. van Gunsteren, A. DiNola, and J. R. Haak, J. Chem. Phys. 813684 (1984).

44. M. Reshchikov, H. Morkoc. J. Appl. Phys., 97, 061301 (2005).

45. E. Wendler, W. Wesch, A.Yu. Azarov, N. Catarino, A. Redondo-Cubero, E. Alves, K. Lorenz, Nucl. Instr.Meth Phys.Res, 307394 (2013)

46. N. Catarino, E. Nogales, N. Franco, V. Darakchieva, S. M. C. Miranda, B. Méndez, E. Alves, J. G. Marques and K. Lorenz, Europhysics Letters 9768004 (2012).

47. A.I. Titov, P.A. Karaseov, A.Yu. Kataev, A.Yu. Azarov, and S.O. Kucheyev, Nucl. Instr. Meth. Phys. Res. B 27780 (2012)

48. A.I. Titov, V.S. Belyakov, A.Yu. Azarov, Nucl. Instr. and Meth. B, 212, 169 (2003).

49. P.A. Karaseov, A.Yu. Azarov, A.I. Titov, S.O. Kucheyev, Semicond. 43691 (2009).

50. P. Ruterana, M. Albrecht, J Neugebauer, Nitride semiconductors: handbook on materials and devices. (John Wiley and Sons, 2006). 